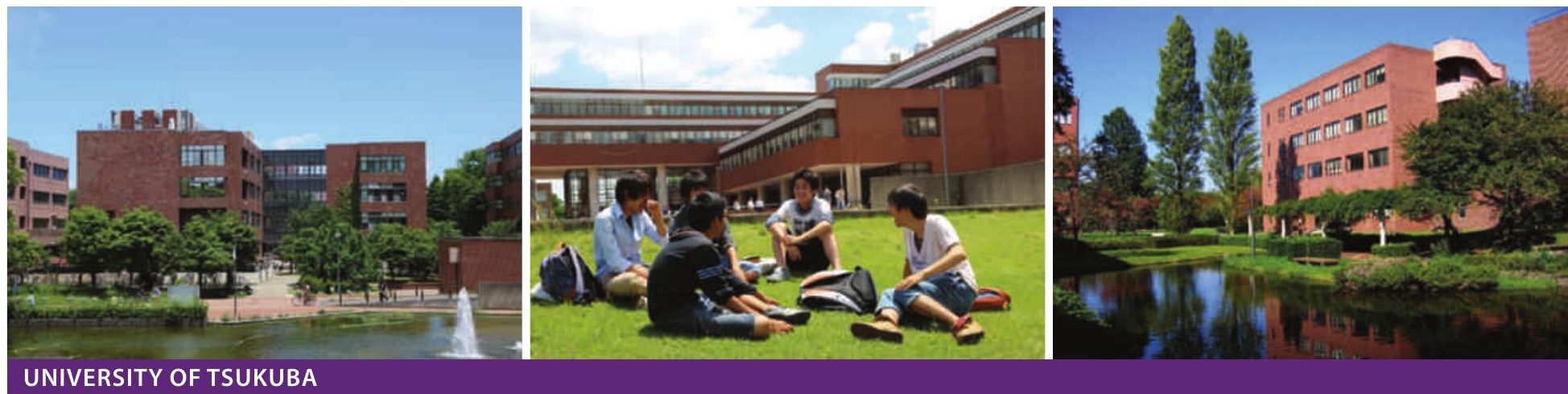

\title{
A world of opportunities
}

\section{$\mathrm{T}$}

ransplanted from Tokyo in the 1970 s to become the heart of Tsukuba Science City, the University of Tsukuba continues to build on its 140-year legacy of progressive and innovative reform of its research and education systems with new globally oriented initiatives that challenge convention.

"Our university, established originally as the Tokyo Higher Normal School in 1872, was Japan's first government higher-education institution," explains Kyosuke Nagata, the ninth president of the university since its transformation into a comprehensive national university in Tsukuba in 1973 from the Tokyo University of Education. "We preceded the University of Tokyo and Kyoto University. We have produced Nobel laureates and also dozens of Olympic medallists who have won a total of 61 medals."

"The institution's founding president Jigoro Kano, the father of judo, believed that the research mind should develop from a healthy body and brain, and promoted sport as a means of achieving academic success," says Nagata. Kano's legacy continues to live on and extend its influence. In Japan, sports scientists are already focusing on the 2020 Tokyo Olympic and Paralympic Games, hoping to improve on Japan's largest medal haul to date-38 medals at the London Olympics in 2012.

The University of Tsukuba has a firm basis of prestigious fundamental research, including world-leading accelerator physics, anthropological research in west Asia, and the study of sleep at the World Premier Research Center
Initiative. Building on such a firm foundation, the university is responding rapidly to the increasing demand for innovation by conducting advanced research into personal care robots and energy production from algae.

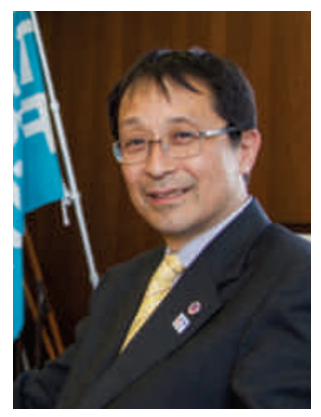

\section{We have innovative research and education systems, which are unique in the world.}

Kyosuke Nagata, President
Design further extends the university's scope.

"Kano was very progressive; even in the early Meiji era in the late 19th century, Kano had the foresight and courage to invite thousands of international students to study at the university," says Nagata. In addition to having a global outlook, the university is also interdisciplinary, playing a central role in Tsukuba Science City, where there is a cluster of national research institutes in various fields. Collaborative graduate schools have been established in partnership with local institutes, fostering ground-breaking interdisciplinary and inter-sector research.

"We are well-ranked internationally as a comprehensive university, particularly among young universities, but we are really focused on innovation in research and education - breaking down the barriers that exist between traditional faculties and departments, and bringing together students and researchers from diverse backgrounds to create something new," explains Nagata.

The internationalization effort of the university is ongoing; this year it established a new scheme that enables young assistant professors to perform research at some of the most prestigious institutes in the world during their tenure-track periods. Another new system introduced this year is one that allows faculties to invite top-level researchers to the university as part-time professors; this has the benefit of exposing graduate students to cutting-edge research.

Tearing down the walls between departments has been a particular goal of Nagata. "In Japan especially, students become tied to a specific faculty or department early in their education, and belong to that department for the rest of their academic careers," notes Nagata. "We want to break that close singlediscipline association. Now, students and professors are associated with undergraduate and graduate schools constructed from a range of disciplines. We have no regular engineering department, for example, but many schools with engineering components. This is how we hope to specialize, to innovate. It is what we want to become valuable for."

\section{University of Tsukuba \\ \#www.tsukuba.ac.jp/english}



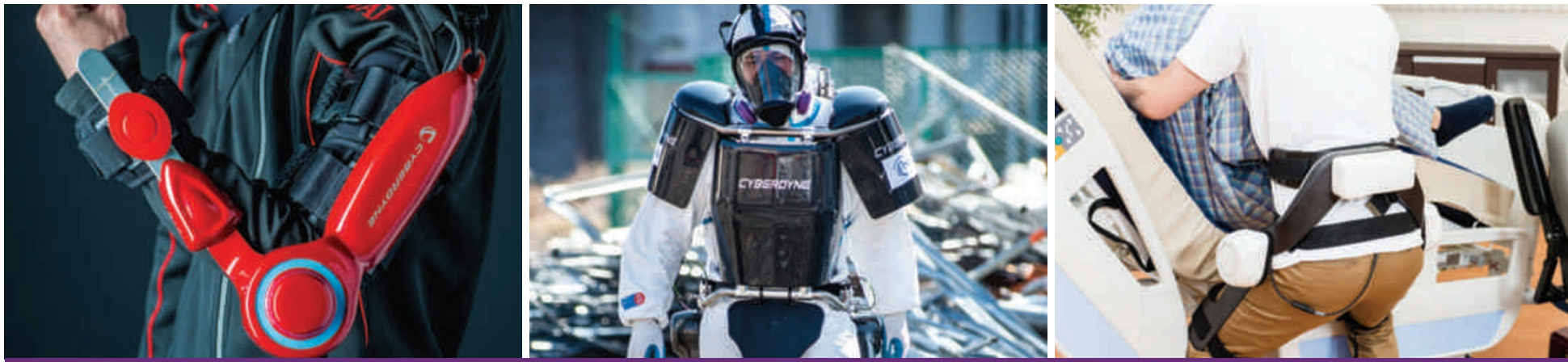

\section{Making the impossible possible}

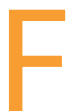

ew concepts provoke more fascination than the fusion of human and machine. It is the stuff of science fiction, of robotic limbs, physical augmentation and neural interfaces. Yet that is the very future imagined — and made a reality — by Yoshiyuki Sankai, director of the University of Tsukuba's Center for Cybernics Research.

"The future will present many different problems for human society," says Sankai. "I believe innovative technology should be created to solve future problems, even if we can't imagine yet how that might be achieved. I began wondering how it might be possible to aid human movement and action by somehow fusing humans and technology. I could imagine the final goal but at that time there were no methods to get there."

Faced with voids between the disparate disciplines needed to develop such technology-neuroscience, physiology, robotics, computer science, information science, medicine and engineering, as well as behavioural science, psychology, ethics, law and science - Sankai devoted his effort to establishing the world's first interdisciplinary academic field of 'cybernics.' "Professors are usually assigned and fixed to traditional fields," he explains. "My vision could not happen within that system, so I decided to establish a new academic frontier."

In those early days some 25 years ago, Sankai and his small team began by exploring the possibilities of cybernic technology, using animals to understand bioelectrical signals

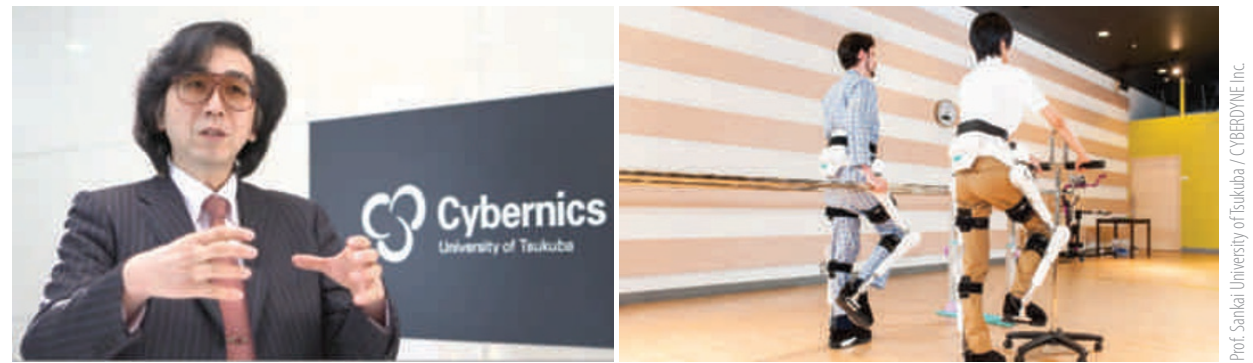

\section{I believe innovative technology should be created to solve future problems. \\ Yoshiyuki Sankai, Professor}

and how they could be utilized artificially. After eight years of prototype development, the centre unveiled its first cybernic device, the Hybrid Assistive Limb (HAL) system.

"HAL-1 had thick electrical cables and some large fixed computers," says Sankai. "The cables were only five metres long, so it was difficult to evaluate the full range of movement, but we demonstrated for the first time that human and robot could work together naturally."

Sankai's technology is based on detecting faint bioelectrical signals that are 'leaked' to the skin surface, and translating those signals into movement. The mechanical parts of the system are programmed to respond to those signals in a natural way. HAL-1 was a first-stage breakthrough, taking the desire to move and translating it into actual motion.

"By 2000 we had developed an independent robot suit, HAL-3, with no cables. Although it was bulky, carrying large batteries and computer systems, we were able to conduct field trials outside," says Sankai.

\section{A plan for the future}

HAL-3 represented a turning point for the centre. After meeting with a famous Japanese manufacturer, it became apparent that there was considerable interest in Sankai's work and that a plan was needed to get the technology out of the laboratory and into real service. "Up until then we had been concentrating on getting the technology working," he says. "But I realized we needed a real plan to turn this technology into commercial devices. That's when I decided to focus on human assistance by making devices to help patients with nerve damage and other mobility problems."

To further the development of commercial HAL models, Sankai established the university venture company Cyberdyne in 2004. Yet just like its founder, the company too is extraordinary by its fundamental nature. "Most companies face challenges like increasing sales or reducing costs," explains Sankai. "We don't even have a market. Our challenge is for the future, so there are no industries, no guidelines, 

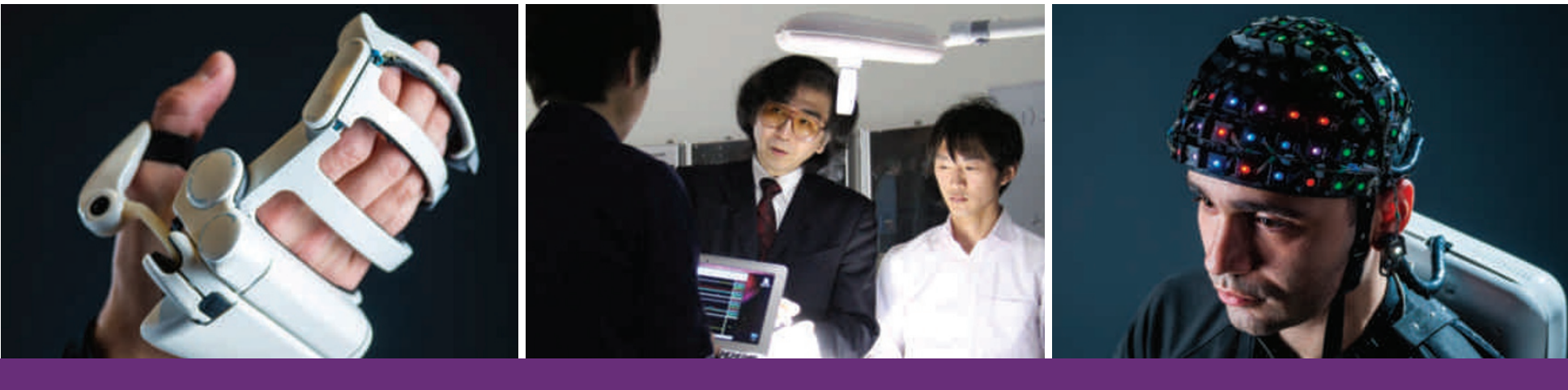

no laws, no professionals and no investors. We need to create it all."

\section{The world's first medical robot}

"Our goal is to restore human function through interactive bio-feedback," says Sankai. "We do this by detecting bioelectrical signals, then adapting, correcting and returning the signal to the patient. This is a medical application which requires ISO certification as with all medical devices. Our problem was that there were no rules or committees under ISO for medical robots. So these too, we had to create."

Sankai himself and members of Cyberdyne became expert members of a new ISO 13485 committee for medical robots, and recently obtained the world's first ISO and CE approvals for such devices. "Our Medical HAL system is now used in clinical trials in Germany for functional improvement therapy such as neurorehabilitation, and the treatment is covered under the German public workers' compensation insurance," says Sankai. "We are now obtaining good clinical data, which we will eventually use to secure full medical approvals for this therapy in order to extend its application to other diseases."

The Medical HAL systems provide neurorehabilitation for patients who have suffered spinal and nerve injuries, including strokes. A total of $29 \mathrm{HAL}$ systems in eight different bodyweight-supported treadmill lanes are now in use at a new centre for robotic rehabilitation at the BG University Hospital Bergmannsheil in Germany - a hospital specializing in cuttingedge technology for the treatment of occupational injuries. There, each suit is used by up to four patients per day, and the results have been so astounding that already another eight hospitals in Germany, including hospitals in Berlin and Frankfurt, are set to start using the therapy routinely towards the end of 2014.

"We have eight operating Medical HAL systems in Germany, and we can imagine systems being deployed to all hospitals in Germany, and possibly throughout Europe," notes Sankai.

\section{Making miracles routine}

The level of innovation embodied in the Medical HAL robot suit has not gone unrecognized. In 2014, this device was awarded gold in the prestigious Edison Awards. Some of the astonishing success stories that have emerged out of the clinical trials in Germany are also rewards in themselves. Like the case of Philippe von Gliszynski, who was paralysed from the waist down after a fall. Conventional wisdom would have had Philippe in a wheelchair for the rest of his life, but after just six months of training using Medical HAL, he was able to walk over a kilometre using only a walking frame.

"Even in cases of severe neurological damage, bioelectrical signals are still produced, and these can be used for therapy," explains Sankai. "We are now looking at combination therapies, using a detection scheme similar to functional magnetic resonance imaging in conjunction with botox or stem cell therapy to restore motor function. For example, botox is routinely used to stop spasms by releasing the synaptic connection between muscles and nerves. After botox treatment, patients have relief but not sufficient ability to move their limbs. We can use cybernic technology to drive their bodies and restore functionality. We have seen that it helps facilitate

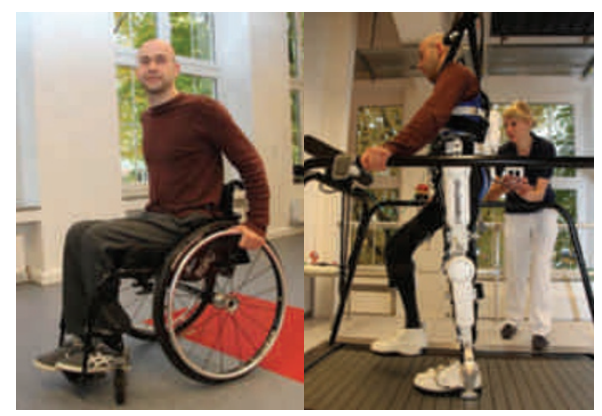

HAL therapy made it possible for a patient paralyzed from the waist down to walk again. nerve connections or reconstruct nerve circuits, even without stem cell therapy. It's a completely new mode of therapy, and very promising."

\section{An innovation spiral}

The nascent success of Cyberdyne is very much tied to the opportunities provided by its establishment in Tsukuba Science City. The Center for Cybernics Research is itself a product of the progressive and experimental mindset of the University of Tsukuba, and together with Cyberdyne promotes an 'innovation spiral' to turn Sankai's imaginings into reality. "The innovation spiral entwines basic research, social-implementation and human resource development simultaneously," explains Sankai.

Over 400 of Cyberdyne's commercial HAL for Wellbeing system, a non-medical model, have already been leased to 170 hospitals in Japan. The feedback has been very promising, and these preliminary trials are providing crucial pre-clinical data for evaluating the system's medical efficiency.

Propelled by this success, the company is now producing various HAL versions, such as single joints, lumbar support and a full-body version for heavy lifting. Various other human assistive technologies are also in development, including vital-sign sensing devices, health care devices and communication support devices.

On the development table right now are robotic leg prostheses, hand and finger versions, and communication and manipulation aids for patients with severe motor function impairment. "We are also using our bioelectrical detection technologies to develop a nonintrusive wearable monitor to pick up the early warning signs of stroke. We are always imagining new ways to use our technology for the benefit of human society."

Center for Cybernics Research

\#ww.first.ccr.tsukuba.ac.jp/english/ 

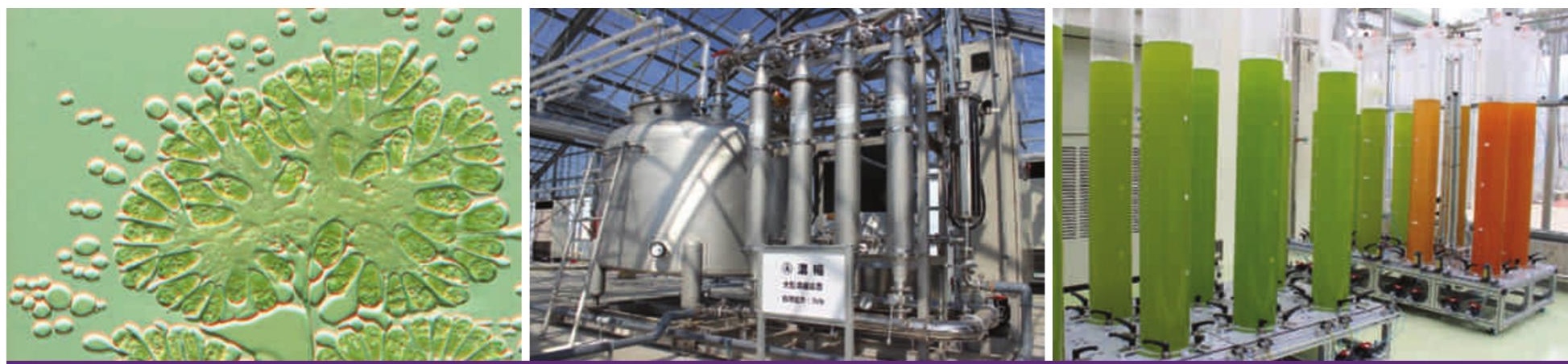

CORE LABORATORY FOR ALGAL BIOMASS AND ENERGY SYSTEM

\section{The future is looking greener}

\section{A} s the world's fossil fuel reserves edge closer to depletion, many scientists are turning their attention to alternative sources of hydrocarbons. Algae are fast becoming the most obvious and attractive future source of such fuels, but the development of algal fuel technologies faces some significant challenges. The University of Tsukuba's Core Laboratory for Algal Biomass and Energy System, Japan's leading centre for algal fuel research, is poised to tackle this global energy challenge head-on.

Sustaining civilization into the future, beyond the limited supply of fossil fuels, will inevitably require the introduction of commercial alternatives to the fuels we use today. There has been a strong push towards using renewable energy sources such as hydro-, wind and solar power, primarily to combat climate change. These renewable energy sources, however, target only electricity supply, while society remains almost inextricably dependent on mobile combustion-based power sources and petroleum-based products such as plastics.

"In Japan, petroleum use is split almost equally between transport and petroleum products," explains Makoto Watanabe, a key member of the laboratory. "Many alternatives for electricity generation already exist, but the demand for petroleum will be very difficult to reduce. We must develop an alternative to petroleum. This is a very big challenge."

Maize and other higher plants are already providing a source of 'bioleum' to supplement petroleum supplies, but this approach, says
Watanabe, can only be a temporary solution. "The cropping of plants for biofuel production competes with agricultural food production and ties up arable land, which is already distorting food prices and availability. And although higher plants and algae both produce oil directly, algae can produce ten to a hundred times more oil per hectare compared to higher plants. Algae are therefore very attractive, and a future 'algal economy' seems unavoidable."

Yet there are some significant obstacles to be overcome before algal bioleum production can hit the mainstream. Most algae and higher plants produce triacylglycerides, which only become usable as a petroleum substitute after chemical conversion to biodiesel - a process that requires dedicated chemical refineries, because biodiesel has a lower oxidative stability than petroleum-derived fuels. Biodiesel also has elevated

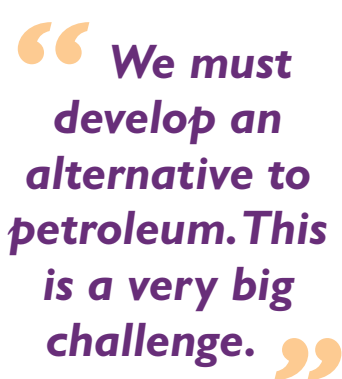

Makoto M. Watanabe Professor braunii, that produces hydrocarbons. Although it produces the 'right oil', this species fails to address the core issues that drive the development of alternative fuels. As an autotrophic or photosynthetic species, it can only be grown in ponds no more than 20 centimetres deep. This means that replacing even a small fraction of Japan's transport fuels with Botryococcusderived biofuels would require many thousands of square kilometres of algal ponds.

Pond area is not the only drawback of Botryococcus, however. It also requires temperatures between 20 and $30^{\circ} \mathrm{C}$ for optimal growth and consumes large amounts of nutrients to produce its harvestable oil. "Japan's climate is generally too cold for Botryococcus," says Watanabe, "and nutrient costs, particularly for inorganic nitrogen and carbon dioxide, are significant."

There is clearly a need to find algal species that address the nitrous oxide emissions and poor cold-weather combustion characteristics.

"The focus is now on 'drop-in' bioleum fuels that can directly replace existing petroleum products as they can use existing refineries and delivery systems and that offer the same combustion characteristics as existing fuels," notes Watanabe. "That means we need to find and develop hydrocarbon-producing algae."

\section{Finding the right match}

Over three decades ago, scientists discovered an autotrophic algal species, Botryococcus shortcomings of Botryococcus. "We were the first to discover that a strain of heterotrophic microalga called Aurantiochytrium mangrovei from Okinawa produces and accumulates hydrocarbons," says Watanabe. "As a heterotrophic alga, it produces its biomass not by photosynthesis but by consuming organic matter. This means it can grow very rapidly, more than 30 times the rate of biomass and hydrocarbon production of Botryococcus, and it can do so in a fermenter tank rather than a shallow pond. Heterotrophic algae are highly advantageous for regions such as Japan and 


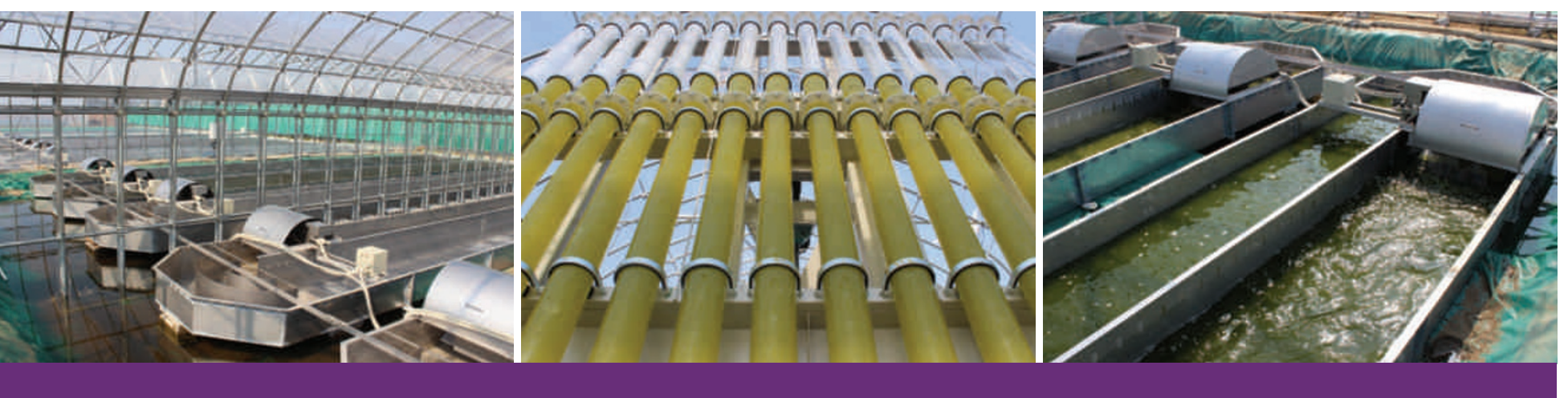

Europe, where annual sun irradiation is low compared with western USA and northwestern Australia."

In addition to addressing many of the problems that have prevented Botryococcus from becoming a commercial technology, Aurantiochytrium produces a purer and more usable hydrocarbon product compared to Botryococcus. Some strains of the alga produce up to $99.7 \%$ squalene, which can be directly refined by conventional hydrocracking to produce jet fuel and diesel oils in yields superior to those from crude oil. This pure squalene product is also useful for cosmetics, food, medicines and pharmaceuticals. The laboratory for algal biomass is soon to add a two-tonne fermentation reactor for Aurantiochytrium to produce the quantities of biomass needed to develop this technology for mass production.

\section{From waste to energy}

"At present, it takes more than 35 gigajoules of energy to produce enough carbon dioxide to generate just 11 gigajoules of botryococcal hydrocarbon," observes Watanabe, "as well as two gigajoules of energy to produce the inorganic nitrogen needed. Cheap nutrients are essential. This is why three years ago we started developing technology to use wastewater streams and the emissions of wastewater treatment plants to provide the energy and nutrients needed for algal fuel production."

The strategy is elegant in its simplicity, albeit technically challenging. Sewage provides the nutrients, while the sludge incinerator provides a source of heat to maintain optimal algal growth temperatures, and gaseous emissions to provide the carbon dioxide needed for photosynthesis. Watanabe's concept includes both Botryococcus and Aurantiochytrium; the autotrophic species taking the secondary wastewater effluent as a source of inorganic nutrients and carbon dioxide emissions, and the heterotrophic species using solubilized organic nutrients extracted from excess sludge. "We believe this could be a new model for algal biomass production," says Watanabe.

\section{Local strains}

Watanabe's colleague Isao Inouye is taking a different approach. "Botryococcus and Aurantiochytrium are not suitable for all climates," he explains, "but every region has its own native populations of algae that are well adapted for the local climate. In 2010, we established a consortium of more than 60 companies with the goal of examining the potential of these native populations for algal biomass production, and have now started a pilot project in the Fukushima area."

The Fukushima NextGeneration Renewable Energy Technology Project aims to achieve an annual algal biomass production rate of more than 30 tonnes per hectare using heat and carbon dioxide emissions from thermal power plants, and sewage and fisheries waste as input nutrients. "A similar strategy is already being used in New Zealand, but so far not cost-effectively," notes Inouye. "We are now constructing our culture ponds and hope to demonstrate the best method for achieving our target biomass production rate this year with a view to commercialization. We hope eventually to spread this technology to other cool regions, like Europe."

The counterpart to this biomass production technology is the means for converting the algae into usable fuel products. "We are also developing hydrothermal liquefaction technology to increase the oil fraction, to produce kerogen from the organic components," says Watanabe. "We cannot control the natural oil production characteristics of these native populations, but with hydrothermal treatment, we could turn almost any algal assemblage into a viable biofuel source."

\section{Unfinished business}

Although Aurantiochytrium is attractive for biofuel production from many perspectives, the latent potential of Botryococcus is still difficult to ignore. "We have installed new outdoor research production facilities to produce large amounts of botryococcal biomass, and we are obtaining higher hydrocarbon purities than anywhere else in the world. Only we can produce enough research biomass to make this possible," says Inouye.

"We have also developed a gene transfer system for Botryococcus," adds Watanabe. "This alga has a hard envelope that has prevented researchers from transferring genes. We were the first in the world to succeed, and we have submitted a patent application for the method. This opens the door for genetic modification, which could lead to much higher rates of hydrocarbon production from this species."

With some significant achievements behind them, all with a fraction of the resources enjoyed by peers in the USA, Watanabe and Inouye are now looking ahead to a coordinated Japanese foray into algal biofuel technology. "Our goal is to establish an Algal Biomass Research Center that can orchestrate the unique advanced algal technologies being developed across Japanese institutions," says Watanabe. "This is a global issue, requiring a global response. We believe Japan can collaborate and contribute at the highest level."

Core Laboratory for Algal Biomass and Energy System

\#algae-biomass-tsukuba.jp/en 

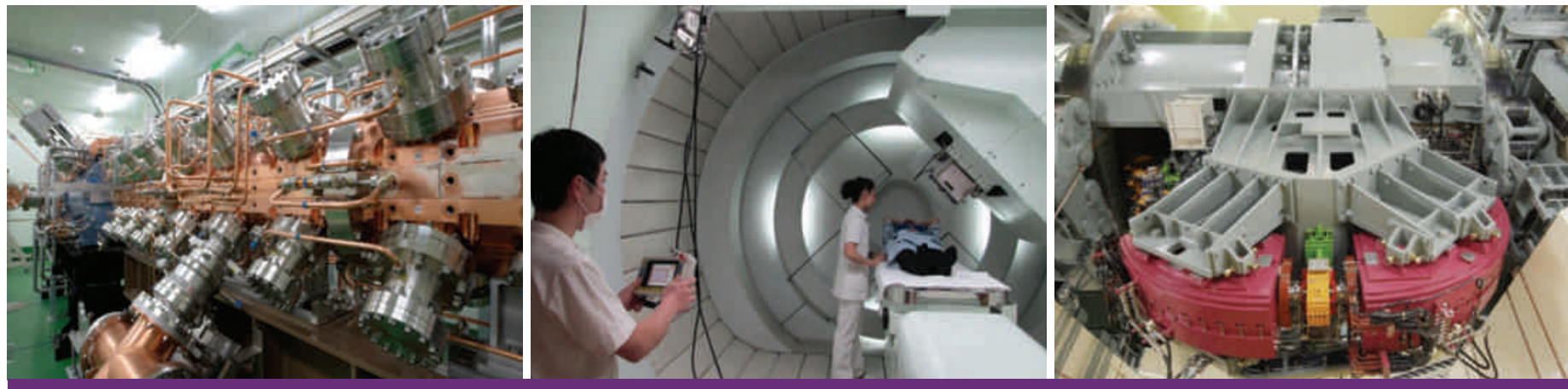

PROTON MEDICAL RESEARCH CENTER

\section{A new frontier in fighting cancer}

\section{- he Proton Medical Research Center (PMRC) at the University of Tsukuba Hospital is one of the few centres in the world where particle physicists work alongside medical doctors and physicians in a clinical setting to develop advanced techniques for cancer treatment. With 30 years' experience in the development and application of proton beam therapy, the PMRC now has its sights on neutron beam therapy and the goal of becoming one of the world's pre-eminent centres for advanced cancer treatment and research.}

Proton beam therapy is one of the most advanced cancer treatments available, allowing highly targeted irradiation of deep organ tumours, such as liver and prostate tumours, with minimal irradiation of healthy tissue. Producing the proton beam for this technique, however, requires particle accelerator facilities that would be more at home in multimilliondollar physics research facilities.

"Our proton beam technology started life at the High Energy Accelerator Research Organization, about ten kilometres from here," says PMRC director Hideyuki Sakurai. "Back then, we used to shuttle patients up to the research facility for treatment. As we made the proton accelerator more compact, it was relocated to this hospital, where we can treat many more patients. We have now treated over 3,000 cancer patients, including 1,200 liver cancer patients with a $90 \%$ cure rate."

The University of Tsukuba's strong ties with the High Energy Accelerator Research
Organization and emphasis on interdisciplinary collaboration has made it possible to continually refine this once prohibitively expensive technology for use in a hospital setting. There are now nine such facilities in Japan.

One of the pivotal technologies that made it possible to apply proton beam technology to cancer therapy safely is that of 'respiratory gating,' where the beam is switched on and off as the patient breathes to ensure that the beam irradiates only the tumour and not the surrounding tissue. "Respiratory gating was developed here and is now used throughout the world," says Sakurai.

Whereas conventional radiation therapy results in high radiation doses to healthy tissue and significant trauma, with patients suffering for many weeks from the therapy's side effects, proton beam therapy is performed for just 30 minutes a day over a two-week period without pain, sedation or anaesthetic. The reduced impact on the body makes this therapy an especially good option for children and the elderly. "We have a very good paediatric cancer team," says Sakurai. "We are also developing a multi-therapy combining targeted chemo and proton therapy, which has proved very effective for advanced lung and oesophageal cancer without the side effects of conventional radiation therapy."

Research by the PMRC's team of physicists and clinicians has led to the development of a new treatment technology - boron neutron capture therapy (BNCT). "Proton beam and conventional X-ray therapies do not cover all cancers," says Hiroaki Kumada, an associate professor at the PMRC. "We are developing this neutron beam technology as a therapy for cancers such as invasive brain tumours, which are difficult to treat by existing methods. Furthermore, BNCT requires just a single treatment session, which is very attractive, particularly for visiting patients."

The BNCT technique was first proposed al most 80 years ago, but the need for a research reactor to produce the neutron beam on which the therapy is based prevented the advancement of this promising treatment. Yet the principle of BNCT is otherwise elegant in its simplicity - a boron-bearing drug that accumulates only in tumour tissue is given to the patient, and the area is then irradiated with low-energy neutrons, which are absorbed by the boron to produce a localized radiation burst that kills tumour cells. As boron occurs at only very low levels in normal tissue, and boron-bearing drugs can cross the blood-brain barrier, BNCT holds much promise as a next-generation cancer therapy.

"We now operate an experimental reactor at the Japan Proton Accelerator Complex 70 kilometres north of Tsukuba," explains Kumada, "and are collaborating on the development of a safe, compact accelerator that could be installed at the hospital. Other countries are also developing this technology, but we have the advantage of working closely with heavy industry to create a commercial device. Our clinical trials start next year, and we hope soon to establish BNCT as an advanced medical care system that can be adopted worldwide."

Proton Medical Research Center

\#ww.pmrc.tsukuba.ac.jp/engindex.html 


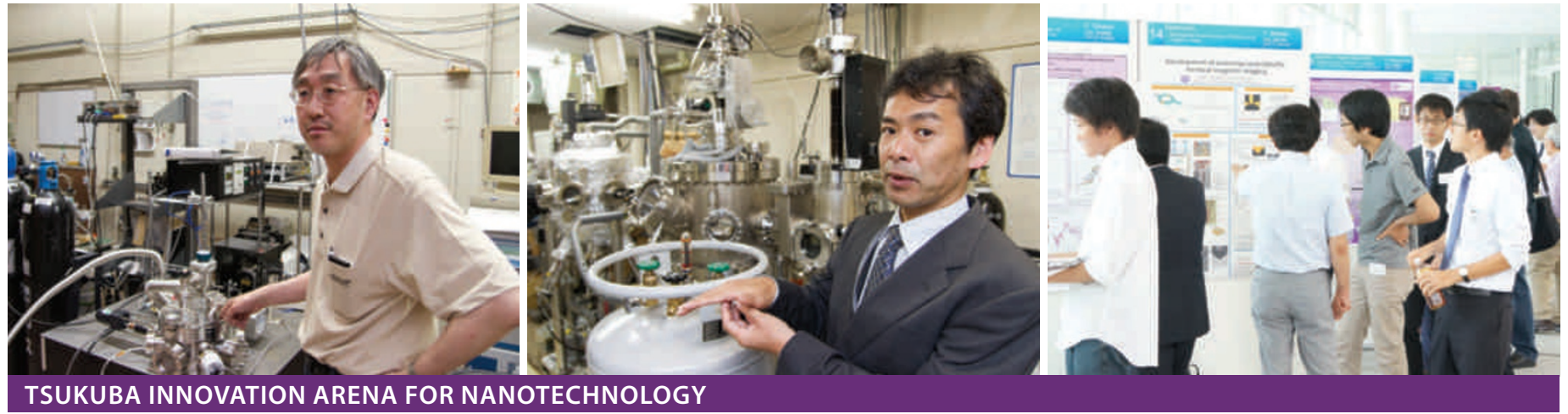

\section{Innovation for the future}

\section{$\mathrm{T}$} he establishment of Tsukuba Science City in the 1970s brought together dozens of national research institutes and hundreds of related industries with the aim of stimulating collaborative innovation in science and technology. The Tsukuba Innovation Arena for Nanotechnology (TIAnano) extracts the most out of this collective expertise by providing opportunities for cooperative research by scientists, engineers and students from a variety of fields.

Innovation often requires a little assistance. Although Tsukuba hosts a critical mass of national research institutes and engineering and technology companies, with the University of Tsukuba at its heart, the tendency for research institutes to work independently has meant that the research and development synergies hoped for through collocation have rarely occurred spontaneously.

In 2009, the university spearheaded the foundation of an 'innovation arena' to forge inter-institute agreements that could nurture the kind of multidisciplinary and multi-sector collaboration needed to make revolutionary advances in nanotechnology. TIA-nano brings together the University of Tsukuba, the National Institute of Advanced Industrial Science and Technology (AIST), the National Institute for Materials Science (NIMS), and the High Energy Accelerator Research Organization (KEK) through reciprocal agreements on the sharing of faculty, students and facilities.

"The goal of the TIA is to integrate the resources in Tsukuba towards establishing a global research centre for nanotechnology," explains Shinji Kuroda, a professor of the University of Tsukuba's Institute of Materials Science and TIA-nano member.

TIA-nano overcomes one of the major obstacles to innovation - developing individuals with the right skill set to allow them to develop the specific technologies being targeted by industry. "Through TIA-nano we set up a human resources development programme where we could fund industry-focused doctoral projects with the institutes," says Kuroda. "Each student is assigned a senior expert with industry experience as a collaboration coordinator alongside the usual academic supervisor and works on the project as part of a collaboration team that includes researchers from participating institutes and industry."

\section{Next-generation electronics}

Kuroda utilizes the reciprocal agreements of TIA-nano to pursue basic research on spintronics. "Spintronics uses an electron's 'spin' instead of charge like in today's electronic circuits, and is considered to be the next generation of electronics," he explains. "Most research is now focused on practical application of this new technology, but there is still much to be discovered through basic research. We are looking into new semiconductor materials consisting of novel combinations of different elements."

Kuroda's team creates new semiconductor materials in their own lab, but the access to the electron microscopy and synchrotron radiation facilities of NIMS and KEK provided by TIA-nano is crucial for probing the nanostructure and atomic properties of these novel compounds.
"Ferromagnetic semiconductors are essential for spintronics, which rely as much on magnetism as electric fields. But most semiconductor materials only become ferromagnetic at very low temperatures. We have found combinations of elements and various impurities that significantly raise the transition temperature, which could make spintronics a practical technology. This is a big discovery, and we are now investigating exactly why these combinations raise the working temperature."

\section{Synergies for energy research}

Another researcher that takes advantage of the collaborative opportunities of TIA-nano is Yutaka Moritomo. "The aim of our laboratory is to create new materials that could be used in new types of devices. We are now looking at nanoporous materials capable of storing charge for next-generation batteries," he says.

Recent research by Moritomo's team focuses on the organic dye molecule Prussian blue. "This molecule has a similar storage capacity to the cathode in existing lithium batteries, but can charge and discharge much faster. We use the facilities at KEK and NIMS through TIA-nano to study the origin of these superior properties. TIA-nano also makes it possible for physicists to apply their knowledge to energy materials, which is very rare in Japan but absolutely essential to further the development of this promising technology."

Tsukuba Innovation Arena for Nanotechnology

\#tia-nano.jp/en/index.html 

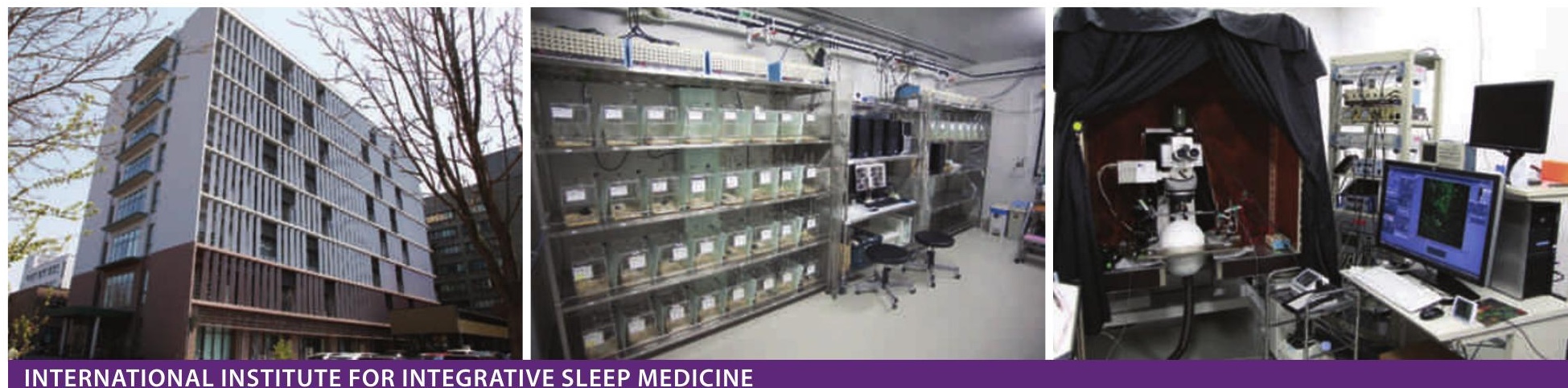

INTERNATIONAL INSTITUTE FOR INTEGRATIVE SLEEP MEDICINE

\section{New strategies to unravel the mystery of sleep}

\begin{abstract}
$\mathrm{T}$ he International Institute for Integrative Sleep Medicine (IIIS) at the University of Tsukuba, one of the nine institutions in Japan's prestigious World Premier International Research Center Initiative (WPI), is setting a new standard for sleep research in Japan, bringing together the best minds and the latest technology to probe the mysterious 'black box' of sleep in new ways.
\end{abstract}

Sleep medicine is one of the most active fields in clinical research, with major sleep centres having been established in the USA and Europe with the aim of developing treatments for sleep disorders such as insomnia and sleep apnea. Sleep disorders have a major impact on productivity and the economy, as well as being a significant risk factor for other lifestyle disorders and one of the most common causes of vehicle accidents and deaths. Yet despite its universal importance to all of us, surprisingly little is known about the fundamental biology of sleep and wakefulness.

This remarkable hole in our knowledge is what drives Masashi Yanagisawa, director of the IIIS. "We aim at really solving the mystery of sleep," he explains. "This is actually unique in the world, because unlike all other major sleep research centres around the world, this centre was not initiated around clinical sleep medicine but around the basic neurobiology of sleep. What we do is still very much a niche area, simply because it is so difficult to study. Sleep is fundamentally a whole-organism phenomenon - you can't do meaningful sleep research in cell culture."

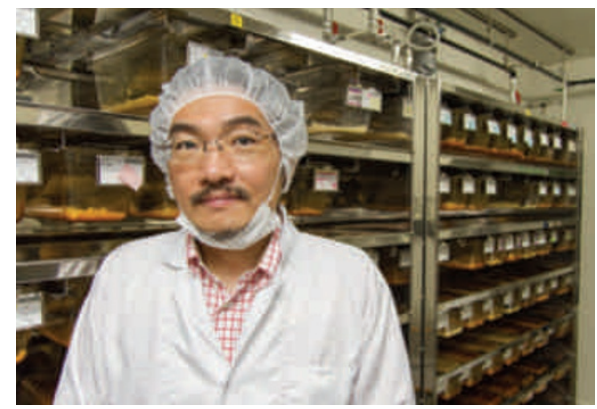

The path to discovery

Yanagisawa's research has taken him from the University of Tsukuba to a distinguished career at the University of Texas Southwestern Medical Center in Dallas, USA. His focus is on molecular intercellular communication, and he describes himself as something of a 'hormone hunter'. His early research at Tsukuba led to the discovery of endothelin, a protein family responsible for regulating aspects of the cardiovascular system. It was in Dallas, however, where his interest in sleep was aroused.

"In 1998, we discovered a neuropeptide called orexin," says Yanagisawa. "It turned out to be a powerful regulator of sleep and wakefulness. Lack of orexin causes narcolepsy, where the brain loses its ability to properly regulate sleep and wakefulness cycles. So this previously mysterious disease could now be explained by a very simple mechanism, the deficiency of just one neuropeptide. After that, my entire lab in Dallas switched to neuroscience and sleep biology, simply because it was so interesting."

Despite spending more than 20 years in the USA, Yanagisawa found the funding on offer in Japan too promising to ignore. In 2010, he

\section{Sleep is fundamentally a whole-organism phenomenon - you can't do meaningful sleep research in cell culture.}

Masashi Yanagisawa, Professor

successfully secured a highly prized FIRST grant from the Japanese government to set up a laboratory at the University of Tsukuba to pursue basic sleep research.

"Orexin itself is not directly usable as a treatment, because the orexin molecule cannot cross the blood-brain barrier to help regulate sleep in the brain," says Yanagisawa. "One of primary aims of the FIRST project was to develop an orexin receptor agonist, something that can activate the sleep-related receptors in place of orexin as a mechanistic treatment for narcolepsy. It was a purely chemical challenge."

The FIRST program provided a major boost to research on an orexin-based drug therapy, and the success of that initiative was the impetus behind the formation of the IIIS WPI centre at the end of 2012. "We were fortunate to get on board an outstanding medicinal chemist, one of the very few outside of industry, and we now have a well-validated molecular target with nanomolar affinity lead compounds. We anticipate having a preclinical drug candidate within a few years, but drug development like this is a long road - it typically takes about 15 years. That is why the WPI funding is so important." 


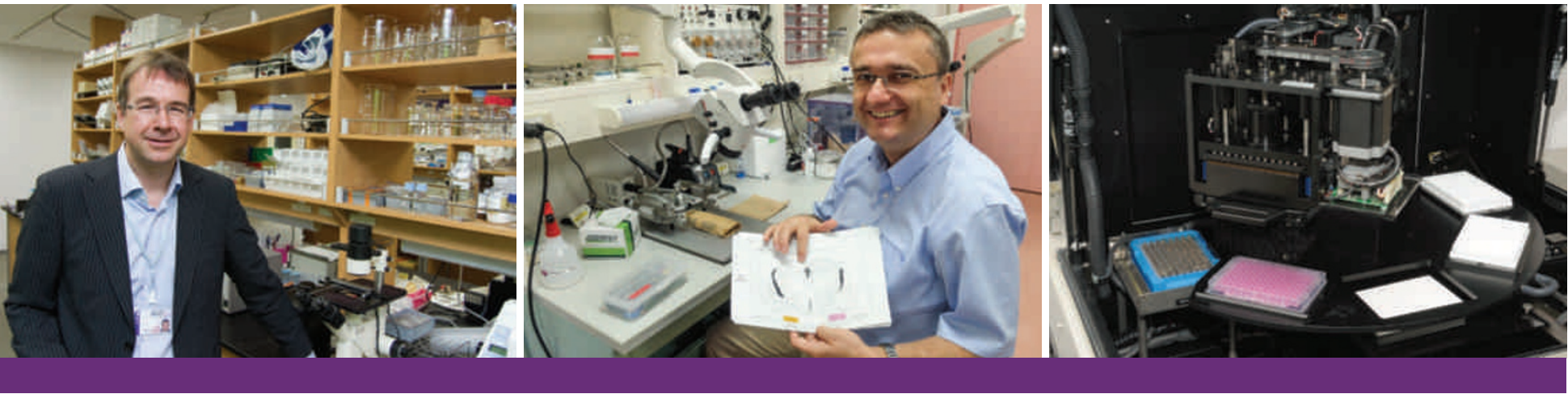

In fact it has taken just that long for the pharmaceutical company Merck to develop an orexin receptor antagonist to assist sleep as a therapy for nighttime insomnia. The drug has recently obtained approval in Japan and the USA, and is to be made available by the end of this year. "This is the first truly new sleep drug in over 50 years, and it is based on our original discovery of orexin," notes Yanagisawa. "At the IIIS, we are creating orexin receptor agonists to assist wakefulness. Besides narcolepsy, such a drug could be applicable to many sleep disorders, such as boosting daytime wakefulness to cure insomnia, as well as treatments for depression and even jet lag. The certainty of WPI funding for at least ten years gives us the opportunity to develop such a drug within academia."

\section{Big science}

The WPI-funded IIIS also continues another major initiative of Yanagisawa's FIRST project - the forward genetic screening of randomly mutagenized mice. "The concept is very simple," explains Yanagisawa. "You randomly mutate the genome of mice using a chemical mutagen, then screen the mutagenized mice for sleep abnormalities. Unfortunately this approach is also very labour intensive. We prepare literally thousands of such mice, and for each individual we measure sleep and wakefulness through electroencephalography and electromyography. It is very laborious, but I decided someone needed to do it. I call it 'big science', and the success and potential of our forward screening program for yielding major breakthroughs in the future was one of the main reasons why we received the WPI funding for the IIIS."

Although only a very small number of the more than 7,000 mice screened to date have displayed interesting sleep or wakefulness disorders, the discoveries made possible by this approach are exciting. The laboratory has now established ten pedigrees with heritable sleep abnormalities, and any one of these could hold the key to a breakthrough in sleep science.

"We have so far identified four new causal genetic mutations for sleep disorders," notes Yanagisawa. "For example, one of the pedigrees — we call it Sleepy - displays hypersomnia. It is awake for only six hours a day, compared to the usual 14. It is the strongest phenotype so far discovered for sleep abnormalities in mice, and we expect that the molecular pathway involving this mutant gene will be the core mechanism for regulating the amount of sleep."

\section{The fascination of sleep}

Having spent so much of his professional research career in the USA, one of the most important things for Yanagisawa in setting up the IIIS was to bring the best aspects of the American research system back to Tsukuba. For example, the institute is able to recruit young and mid-career principal investigators as independent researchers.

One such researcher is Michael Lazarus (central photograph on this page), originally from Germany, who joined the IIIS as founding faculty in 2013. "It was a great honour and a tremendous opportunity," he says. "Here, we have faculty to rival Harvard, as well as cutting-edge equipment, like optogenetics, electrophysiology, deep-brain microscopy and sophisticated sleep recording systems. To find all those things in one place is extraordinary."

Lazarus applies techniques such as deepbrain microscopy using optical fibres inserted into specific brain regions to investigate how the structure of the brain translates into sleep regulation. One part of the brain that is of particular interest to Lazarus is the nucleus accumbens - a brain component traditionally associated with drug addiction and behaviour. "However, we have found that the molecule adenosine, which we know to be involved in sleep as the receptor that is blocked by the stimulant caffeine, is found in the nucleus accumbens. Adenosine in this brain region steadily increases during periods of wakefulness, and eventually makes us so tired that we fall asleep. We are working to visualize this 'molecular' sleep deep in the brain."

Kaspar Vogt (left photograph on this page) is another independent principal investigator who joined the IIIS from overseas; he came to IIIS in 2014 from Switzerland. "I find many aspects of sleep interesting, but what really fascinates me is the way that as we go from wakefulness to sleep, our brain switches to a completely different mode of operation," says Vogt. "During wakefulness, the cortex shows a de-synchronized pattern of activity, probably owing to the many things that are processed in parallel. As we fall asleep, the activity of cortical neurons becomes more and more synchronized and they enter a bistable mode, oscillating between a depolarized and active up-state and a hyperpolarized and silent downstate. Why? What drives this change and what is its benefit? What are the neurons doing? This has been looked at for a long time, but we now have completely different tools for the task."

Vogt's research focuses on the use of techniques such as optogenetics and voltagesensitive fluorescent indicators to observe and control neural activity and networks within the brain with millisecond precision. "In optogenetics, we use light to activate specific neurons that have been genetically engineered to be light activated. It allows us to observe and control the neural network during activity or sleep without pharmacological agents, which was previously very difficult. These techniques will very soon be giving answers to some of the most exciting open questions in the biology of sleep."

International Institute for Integrative

Sleep Medicine

wpi-iiis.tsukuba.ac.jp 

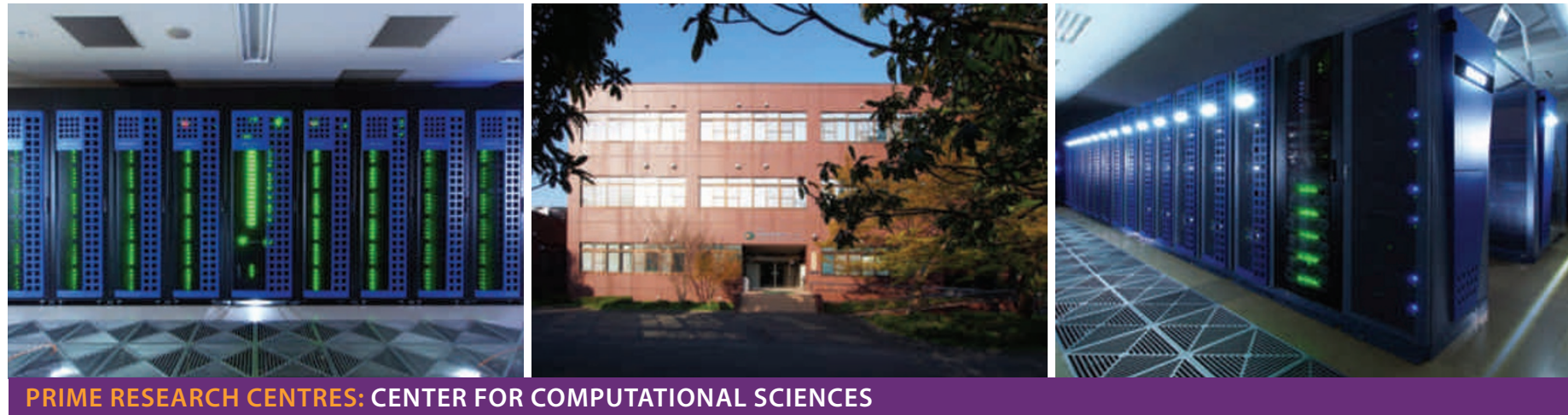

PRIME RESEARCH CENTRES: CENTER FOR COMPUTATIONAL SCIENCES

\section{Parallel goals}

\section{W}

ith over 35 years of supercomputer development behind it, the Center for Computational Sciences (CCS) continues to drive innovation in supercomputer architecture sideby-side with the very scientists who use the machines for research.

It takes a remarkable team of computational scientists and engineers to build a supercomputer. Each new machine architecture involves a complete redesign from the bottom up, a process that typically takes many years and many millions of dollars. Once built, the supercomputer gets only a fleeting chance at being a world-beater. Although the competition for the world's 'fastest' computer is fierce, the primary purpose of supercomputer development is to satisfy the demand for computational resources from research scientists.

"Supercomputer development was started here in 1978 by particle physicists who needed a platform for their simulations," says Masayuki Umemura, director of the CCS. This team of physicists and computational scientists went on to develop a series of supercomputers known as the 'PACS' series. In 1996, after almost 20 years of development, the team achieved the holy grail of computational science with CP-PACS (Computational Physics by Parallel Array Computer System) - the fastest supercomputer in the world at the time.

"CP-PACS was also led by particle physics research, in which we were very strong. That supercomputer was a defining moment for this university and Japanese computational science," says Umemura.

In 2004, the CCS was reorganized into its current multidisciplinary form, taking in scientists from many other departments to collaborate on the development of supercomputers for research. "We have professorships for all of our seven divisions: particle physics, astro- and nuclear physics, condensed-matter physics, life science, global environmental science, computational science and computational informatics. We maintain close collaborations and are all involved in supercomputer development as well as research," explains Umemura. "This allows us to optimize the machines and our algorithms for our research, for which we have been recognized with two Gordon Bell prizes for excellence in parallel computing: one for peak performance in atomic state simulations of a silicon nanowire, and another for the largest-scale simulation of cosmic dark matter."

As supercomputers become more powerful, simulations are becoming an ever more important component of research in many fields, bridging the gap between theory and experimental observation. Competition among researchers for computing resources continues to increase, meaning new and faster computing facilities are always in development.

"We commissioned the T2K-Tsukuba supercomputer in 2008 as a joint project with the University of Tokyo and Kyoto University," says Umemura. By Tsukuba standards, however, T2K-Tsukuba is a somewhat 'conventional' largescale PC cluster. In contrast, the CCS's other supercomputer, the petaflop-class HA-PACS (right photograph on this page), is anything but conventional. "HA-PACS was designed here and is based on more efficient graphics processing units, coupled with other processing units by a new 'tightly coupled accelerators' architecture. Our long history of supercomputer development is very important for designing new machines - our development cycle continues to get shorter."

In recognition of its expertise and facilities, the CCS was selected by the Japanese government as the core centre, Advanced Interdisciplinary Computational Science Collaboration Initiative (AISCI). This gives the centre access to considerable funding, which has allowed it to install yet another petaflop-class machine, COMA (left photograph on this page). "Our goal is to be a world-class centre of excellence for computational science," says Umemura.

The defining characteristic of the CCS is its integration of research and computational science. The centre offers a simultaneous dualdegree program consisting of a PhD in scientific research and a master's degree in computational science. The CCS is also working to establish a Master of Computational Science.

"We are always looking to the future, towards the next-generation, exa-scale supercomputer, and the research that such a computer will make possible," says Umemura. "The CCS is a unique centre for supercomputer development. Whereas others rent computer time, our scientists and engineers co-design and build facilities right here. This is our core vision."

Center for Computational Sciences

Www.ccs.tsukuba.ac.jp/eng/ 


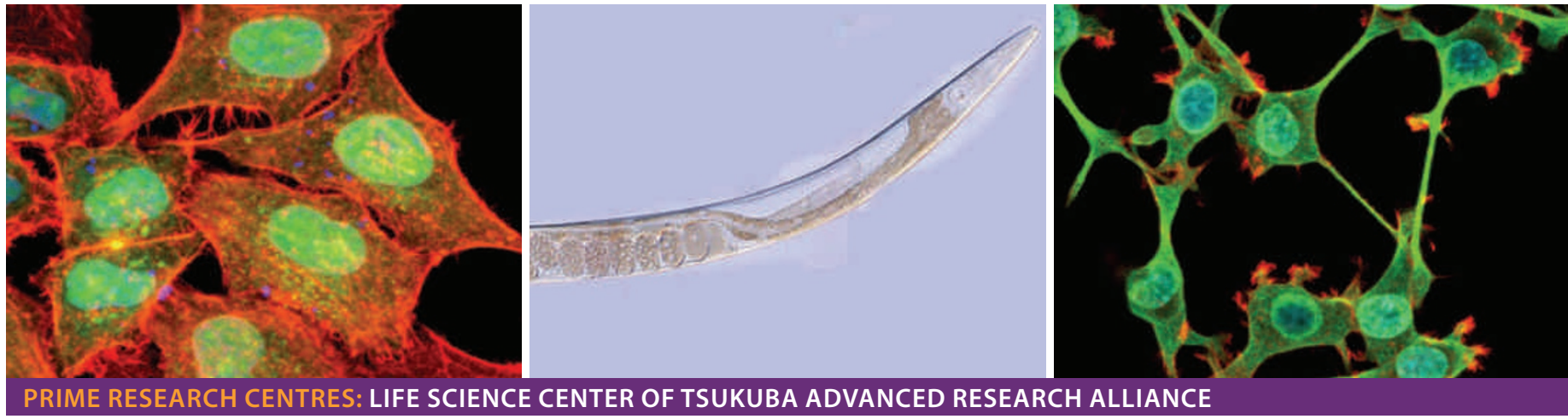

\section{Allies in life science research}

\section{- he Tsukuba Advanced Research Alliance (TARA) was originally established 20 years ago as an inter- disciplinary research centre to promote the most advanced research using the best minds at the University of Tsukuba. In 2010, TARA's mission was changed to focus solely on advanced life science research. It is now one of the university's top-performing research centres.}

"When TARA was restructured in 2010, we needed to choose a single discipline on which to focus," explains Akiyoshi Fukamizu, vice director of the Life Science Center of TARA (LS-TARA). "We had significant government funding, but following a change in policy we needed to simplify what we do. We excelled in many areas, but the potential applications of life sciences were too promising to ignore. In the end it was an easy decision."

\section{The many roles of protein regulation}

Fukamizu's own research is focused on unravelling the complex web of interactions associated with a protein modification mechanism called arginine methylation.

"The activity of proteins in living organisms is regulated by molecular modification," explains Fukamizu. "The addition of a methyl group, or methylation, is one such mechanism. Methylation is catalysed by enzymes at specific sites in proteins. My group has been studying the methylation of the amino acid arginine."

Arginine methylation is catalysed by enzymes called methyltransferases. "The most important of these is PRMT1, which is involved in $85 \%$ of arginine methylation reactions," notes Fukamizu. PRMT1 has many functions. For example, once methylated proteins are degraded, free methylated arginine is released. Unmethylated arginine is involved in the production of nitric oxide, which is one of the most powerful suppressors of blood pressure; arginine methylation inhibits the production of nitric oxide, and therefore may contribute to hypertension. "Finding a way to block the action of arginine methyltransferases like PRMT1 is therefore a potential treatment for high blood pressure, although targeting arginine methylation alone without affecting other critical methylation processes is difficult."

Fukamizu's team is using the model organism Caenorhabditis elegans, a tiny roundworm with a well-studied genome, to examine arginine methylation and the role of PRMT- 1 in more detail. "Other groups have attempted to use PRMT1 knockout mice, but they have early embryonic mortality and provide limited information," continues Fukamizu. "PRMT-1 knockout mutants of $C$. elegans have very short lifespans, which tells us that this methytransferase is involved in longevity. We are now studying conditional knockout mutants to understand the molecular mechanisms of lifespan. It's very exciting research."

\section{Finessing the immune response}

One of Fukamizu's colleagues at LS-TARA, Akira Shibuya, is investigating a range of molecular mechanisms related to immunity. "My laboratory is interested in finding ways to control immune response by regulating immune cell activation and deactivation," explains Shibuya. "Specifically, we are studying various immune receptors, which are expressed on the surface of immune cells and are very important for receiving information from the cell's environment and regulating immune response. We have discovered three receptors - DNAM-1, MAIR-I and Allergen-1 - that are involved in several immune system diseases, such as cancers, allergies and autoimmune diseases."

Shibuya's team has developed a molecular antibody that selectively inactivates these immunoreceptors and have shown that antibody injection into mice suppresses graft rejection and graft versus host disease - common complications of organ and tissue transplant surgery. "For the same reason, DNAM-1 helps to inhibit tumour development. We are now collaborating with a pharmaceutical company to develop an antibody drug for this receptor," says Shibuya.

The other two immune receptors studied by Shibuya's laboratory, MAIR-I and Allergen-1, are similarly promising. "We have obtained evidence that molecular antibody for MAIR-I could be used to suppress dermatitis and allergies, while agonistic antibodies that stimulate the antigen to Allergen-1 suppress anaphylaxis and asthma," says Shibuya. "We were the first to identify many of these molecules and ligands, and in this area we lead the world."

Life Science Center of Tsukuba Advanced Research Alliance

(www.tara.tsukuba.ac.jp/indexENGLISH.html 


\section{Mega-study reveals the Asian roots of civil society}

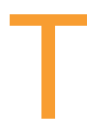
he concept of civil society, where the concerns of citizens are promoted through interest-based associations, is regarded in Asian countries as a particularly Western idea. Yet political scientists agree that such activities form the basis for the democratization of society. "Civil society is the key to solidifying democracy," says Yutaka Tsujinaka, a professor of political science and director of the Institute of Comparative Research in Human and Social Sciences, "a way of embedding democracy in daily life. That is why external democratization often

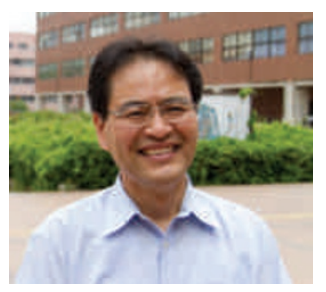

fails - it must originate from within, based on existing community structures."

Understanding the process of democratization is particularly important in Asia, where in many countries true democracy has yet to take hold. Even in Japan, which has upheld a stable democracy for almost 70 years, grassroots democracy in the sense understood by Western political scientists remains elusive.

"In Asia, there is a different social fabric not captured by external surveys - the community or neighbourhood association," explains Tsujinaka. "Although not regarded as civil society, it represents very strong social capital that could be leveraged for democratization if we can fully grasp its significance. That is why we started a comparative 'mega-study' of Asian society."
Yutaka Tsujinaka, Professor
Whereas most studies on civil society rely on census data, Tsujinaka's project aims to directly survey group leaders to gain a more comprehensive understanding of community structures. So far, his team has collected more than 60,000 surveys from participants in 15 countries in Asia and Europe, as well as the United States. "We have also surveyed about 30,000 of the 300,000 neighbourhood associations in Japan, and we will continue to collect data. Surveys are not usually conducted for such studies, but we are trying to capture the civil structure as a whole."

In Japan and other Asian countries, local festivals, events and customs are part of everyday life. It is easy to forget the importance of such community activities and the bonds they form, but their true significance becomes apparent in times of need. "Community and social capital comes to the fore in disasters, such as Japan's recent devastating disasters in the Tohoku region. I have written many books on the subject, but even I was surprised at and reassured by the strength of our community groups at that time."

Faculty of Humanities and Social Sciences \$www.jinsha.tsukuba.ac.jp/about

\section{Open standards and the creation of a global business ecosystem}

\section{Creating a business ecosystem is very important in the current business environment.}

F rom ubiquitous USB plugs to computer operating systems that allow digital mobility across makes and models of smartphones and computers, open standards have created a globally interconnected electronics market. These standards foster the development of a complex business ecosystem involving device manufacturers, materials suppliers, content producers and consumers, all of which Hirofumi Tatsumoto, an associate professor at the Graduate School of Business Sciences, is trying to understand.

Using case studies of specific technologies, such as personal computers or mobile phones, Tatsumoto has analysed how the introduction of standards creates new opportunities in emerging economies, which in turn leads to drastic price cuts and popularization of technologies.

Yet the tale of open standards has not always ended happily for Japanese companies.

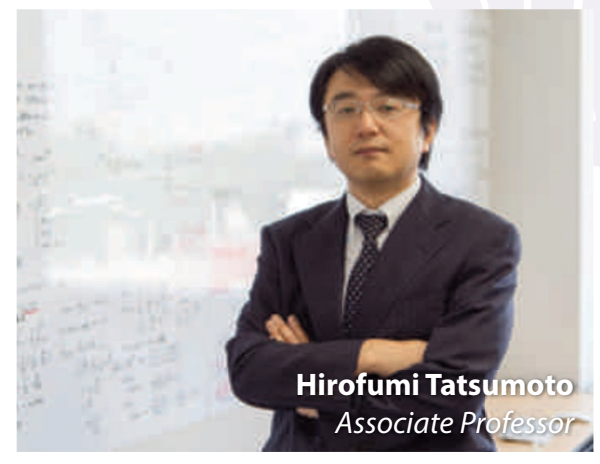

In 1997, for example, an international DVD standard brought about a proliferation of DVD products worldwide, but it weakened Japan's dominance over the industry and assisted Chinese DVD-player manufacturers and content producers in the United States. Japan's flatpanel display industry suffered a similar fate in the early 2000s. Furthermore, Tatsumoto's prognosis for the newest ultrahigh-definition flat panels is no less grim.

"Creating a business ecosystem is very important in the current business environment, but the concept is still very new to Japanese companies," he explains. "Unlike 20 years ago, when the industry was still playing catch-up, Japanese companies today need to generate new markets, and open standards will have to be an important part of that process." Tatsumoto uses his research to strategically advise leading Japanese companies and ensure their global competitiveness under the new rules. And with the University of Tsukuba's strong industry links, his research may eventually add a positive twist to the story of open standards in Japan.

Faculty of Business Sciences

\$www.gsbs.tsukuba.ac.jp/english 


\section{The keys to the Universe}

F ew research endeavours have involved as much investment and collective expertise as the search for the Higgs boson. The final ingredient of the Standard Model of particle physics, the Higgs boson was first theorized 50 years ago but was only experimentally observed in 2012 following a monumental international research effort using the Large Hadron Collider particle accelerator in Europe - considered the most complex and costly scientific instrument ever constructed. Such is the importance of'the Higgs'to physics.

"The Higgs boson holds the key to advancing particles physics," notes Fumihiko Ukegawa, a particle physicist and professor at the University of Tsukuba's Institute of Physics. "It is the origin of mass, and it took many years and an international collaboration of more than 3,000 physicists to find."

The institute was a key participant in the search for the Higgs boson, contributing through the co-development and operation of a silicon-based particle tracker, as well as analysing the data.

The institute is involved in many leading studies in nuclear and particle physics, including collaborative experiments at Brookhaven National Laboratory and the recently decommissioned Fermilab Tevatron in the United States.

"With the discovery of the Higgs boson, we enter a new era of particles physics. Although the Standard Model is now essentially complete, we continue to search for hints of new laws of particle physics - that's when it gets interesting."

The institute is also developing special satellite-borne detectors to investigate cosmic neutrinos. "Neutrinos have very small mass, many orders of magnitude smaller than other elementary particles, so maybe they too hold some secrets," smiles Ukegawa. "In our lab, we are developing detectors sensitive enough to observe them.

"We are also contributing to the development of Japan's radio observatory on a plateau

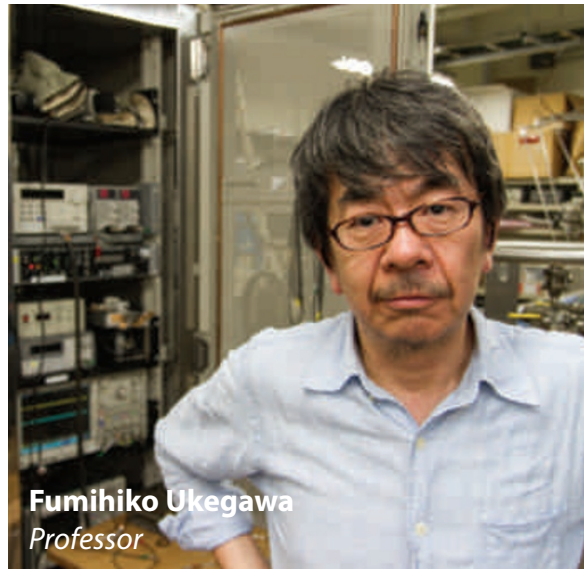

The Institute of Physics was a key participant in the international search for the Higgs boson at the Large Hadron Collider in Europe.

in Antarctica, which we hope to use to analyse the first generation of stars and galaxies that were formed after the Big Bang. Our goal: to understand the Universe and its history."

Faculty of Pure and Applied Science

\#www.pas.tsukuba.ac.jp/ pas-f/english/

\section{Computer-assisted design pushes the limits of origami}

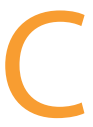

omputer graphics is an unusual world somewhere between art, engineering and science, and the talented individuals that work in this field have virtual feet in each of these disciplines. Few, however, make the transition from the virtual world to something more tangible. For Jun Mitani, whose desk is cluttered with very real but seemingly impossible origami pieces, this translation from the virtual to the real has been a driving

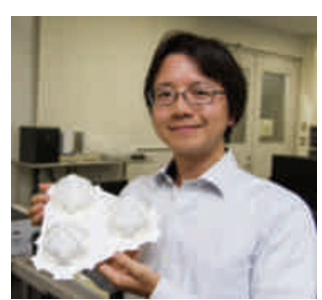

Jun Mitani

Associate Professor
We are developing design tools to help even non-technical users create whatever they can imagine. force for his work since graduating from the University of Tokyo.

"As an undergraduate, I studied precision machine engineering, computer graphics and computational geometry," says Mitani. "In computer graphics, we are completely free - we can make anything we can imagine. In the real world, there are many physical constraints."

Mitani started with origami as an inexpensive but elegant way to explore the application of computational geometry to the creation of real objects within very strict design constraints. With a single sheet of paper, he has managed to design some remarkable origami pieces, including spherical and other highly challenging shapes.

"Although the mathematics of origami has progressed, actual design techniques have not.
I want to explore the boundaries of what can be made with a single sheet of paper and have developed an interactive design tool for non-specialists so that anyone can try origami design. In this way, I hope to extend the origami tradition and highly constrained design techniques to industrial design."

Three-dimensional (3D) printing is another area where Mitani's origami-inspired design principles are a natural fit. "As 3D printers become more affordable, users need interac tive design tools to help them create the objects they need," says Mitani. "We are developing design tools to help even non-technical users create whatever they can imagine."

The University of Tsukuba hosts one of Japan's largest computer science communities. Supported by this community's culture of collaboration and exploration, Mitani has found the ideal environment in which to test the limits of Japan's traditional art of origami and break new ground in computer-assisted design.

Faculty of Engineering,

Information and Systems

www.sie.tsukuba.ac.jp/english 


\section{A nexus for Earth and environmental studies}

W hen 'Tsukuba Science City' was founded in the 1970s, the University of Tsukuba was established by the government as the core collaborative research and educational body, coordinating with the city's national research institutes.

"Under this arrangement, we share researchers and resources with the other institutes in Tsukuba," says Yoshihiro Shiraiwa, a plant physiologist and provost of the Faculty of Life and Environmental Sciences.

The faculty brings together the usually segregated fields of geoscience, biology and agriculture to foster a rare interdisciplinary focus on environmental research that is augmented by collaboration with external research institutes. The university's position as a national leader in the field was highlighted by its selection by the government to be responsible for four strategic organisms under the National
Bioresource Project. "We maintain large collections of tomatoes, microalgae, slime mould, and the sea vase Ciona intestinalis, and our research on these organisms is recognized at the top international level," says Shiraiwa.

Research on microalgae is a particular strength, aided by large algal collections both on campus and at the nearby National Institute of Environmental Studies. "We have many algal scientists," says Shiraiwa, "and a long history of leading research in algal biology, physiology and biochemistry, as well as algal biofuels."

Research into climate change and water is also prominent in the faculty. "We have scientists contributing to the Intergovernmental Panel on Climate Change, as lead authors of the report," explains Shiraiwa. "We also conduct research on weather systems, water management and treatment, and drinking water, with collaborations in Mongolia, China and Tibet."

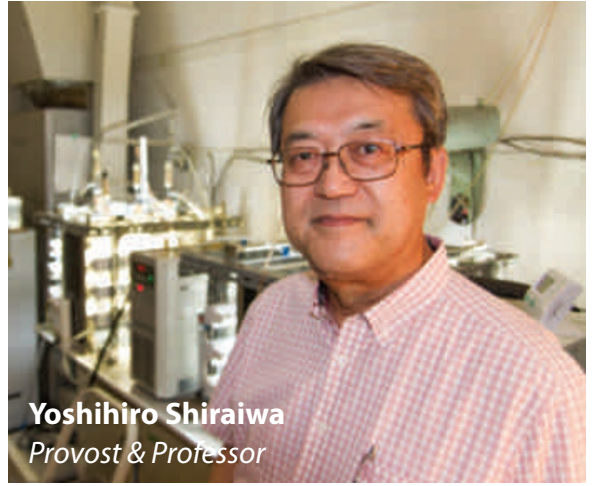

Algal biofuel production is a key area of research in the Faculty of Life and Environmental Sciences.

Environmental science encompasses a broad range of studies, both physical and biological, which are brought together to great effect at the University of Tsukuba. "We may not be medical doctors, but as environmental scientists, we have a key role to play in managing the health of the Earth," notes Shiraiwa.

Faculty of Life and Environmental Sciences \#ww.life.tsukuba.ac.jp/en/index.html

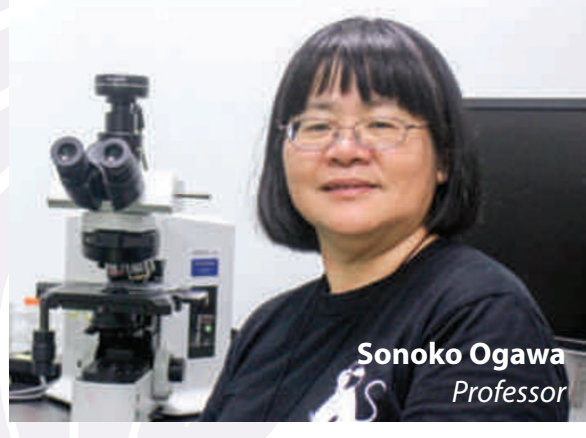

active in fostering a worldwide network of collaborators through the Japanese Society for Behavioral Neuroendocrinology. "We hold and support regular workshops and congresses in our field, with recent events in China, the USA and here in Tsukuba." Yet Ogawa is also keen to emphasize the field's broad significance. "Although it's just basic research now, looking at how hormone action in the brain regulates social behaviour will definitely benefit society in the future."

arating newborn mice from their mothers for even very short periods can have permanent and unexpected social behavioural effects.

"Our research community is still quite small and our main focus here is to train more specialists in this field," says Ogawa, who has been
Faculty of Human Sciences

\#www.human.tsukuba.ac.jp/english 


\section{Leading from the front}

"S tudy hard, train hard. That is the University of Tsukuba tradition," proclaims Hideaki Soya, head of the Human High Performance (HHP) Research Project. This tradition originates with the university's first president, Jigoro Kano, who not only famously founded the martial art of judo, but left a lasting legacy of 'mind and body' that has since resonated around the world. "We of course have many judo athletes, including world champions, but they all study in our graduate school, and some of them are studying for PhDs."

Over the years, the university has produced numerous Olympic and Paralympic athletes, and with 61 Olympic medals and more than 100 medals in Paralympic games, no small measure of success. "This university is worldrenowned for both its sporting achievements and strength in sports science," continues Soya. "We lead the field in many areas, and that is why we have been successful in securing funding for the BAMIS and HHP projects."
The recently concluded Body and Mind Integrated Science (BAMIS) Center was one of the few sports-oriented research centres in the world to focus on both the mental as well as physical aspects of athletic performance, and it produced some important findings.

"We showed for the first time that even mild exercise, like yoga or tai chi, for as little as ten minutes a day actually stimulates the brain, increasing cognitive function. This is a very important result." (This finding was reported in National Geographic, May 7, 2014.)

The HHP builds on the success of BAMIS and expands this investigation even further. "Through the HHP we aim to maximize human abilities like endurance, strength and coordination, but also psychological aspects such as stress resistance," says Soya.

The project has already made an impact globally with the discovery of the role of brain glycogen in physical and mental performance. Brain glycogen, a type of glucose polymer, was

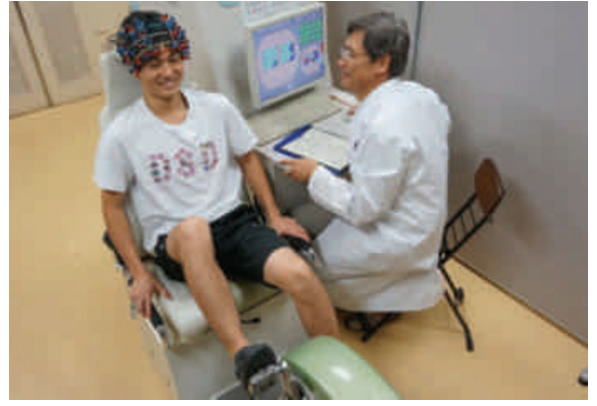

Mild exercise enhances cognitive performance.

thought to be merely an 'emergency' energy source for neurons in the brain, but its function was essentially unknown.

"We found that brain glycogen decreases with prolonged exercise. Is this a form of endurance fatigue? How does carbohydrate loading affect brain glycogen levels? Our current studies show that inhibiting glycogen metabolism reduces endurance, and in mice severely inhibits learning. Glycogen is thus a crucial fuel for both sports and academic performance. (This finding was reported in New York Times, February 22, 2012)."

\section{Faculty of Health and Sport Sciences} \#www.taiiku.tsukuba.ac.jp/english.html

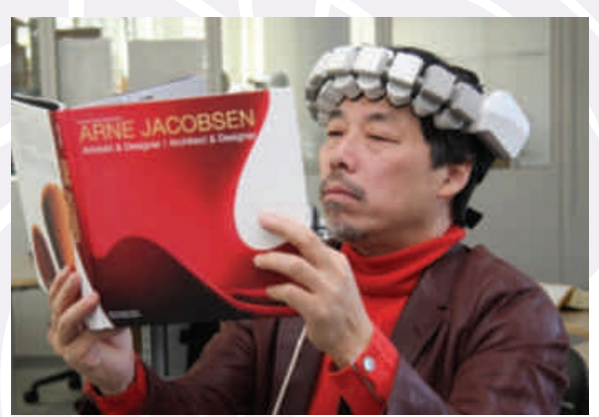

Demonstrating wearable NIRS system.

strategy is now used by product designers throughout the world, but it is applied without a proper understanding of how kansei works on a physiological or psychological level.

"It has taken many years, but we now have a testable definition," says Yamanaka. "We believe that kansei is the process that occurs before conscious thought, prior to cognition - a convolution of our experience that influences our perception of a design and frames subsequent thought and decision-making. It is actually an important brain science process."

The Faculty of Art and Design is the only design school in the world where this notion and its study form the core and guiding principle for design and creativity. "Understanding why and how kansei works can improve the design process," explains Yamanaka. "Kansei can be very powerful. For example, our research has shown that familiarity induces pleasure, but not excitement. Both are needed for good design, so kansei tells us that for good design we need familiar elements but a novel composition that induces excitement. Creativity is still a mysterious process of the mind - I believe kansei is a core part of that process."

\section{Faculty of Art and Design}

-www.geijutsu.tsukuba.ac.jp/english a Mazda coupe unveiled

at the Detroit Motor Show
kansei is a core part of that process.




\section{Untangling a complex genetic web for cancer research}

$T$ he University of Tsukuba has a wellearned reputation in Japan for producing outstanding medical doctors. Yet it is also rapidly building a reputation for worldleading medical research, particularly in the field of cancer biology.

Recent discoveries by Tsukuba researchers in this area have cast a spotlight on the faculty's work. One such discovery was the identification of a strong link between mutations on a gene known as RHOA and an aggressive form of blood cancer called angioimmunoblastic T-cell lymphoma (AITL).

"We found a genetic mutation called $R H O A$ G17V in about 70\% of the AITL cases we studied," says Shigeru Chiba, a professor in the Department of Hematology. "The protein RHOA encoded by this gene has been very well studied and is known to be an important signalling molecule for amoebic cellular movement. It has long been considered a key promoter of cancer invasion and metastasis, but the genetic basis for this role was previously unknown."

Curiously, however, the molecular mechanism of RHOA function may suggest a reversal of the accepted role of this protein. "RHOA normally binds GTP, which is a nucleoside that plays a key role in many subcellular signalling pathways. The mutation prevents RHOA from binding GTP, but how does this loss of function promote cancer? The mutation produces a 'dominant negative' signal, which might mean that in those without the mutation, RHOA may actually act as a tumour suppressor."

Under closer scrutiny, the genetic basis for AITL becomes even more intriguing: an even higher proportion of AITL patients also have inactivating mutations on a gene known as TET2. The protein encoded by this gene is a known epigenetic regulator, meaning that it

\section{Micromanaging crowd intelligence}

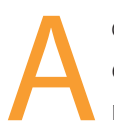

dvances in artificial intelligence have enabled machines to simulate evermore complex forms of human behavior. But some seemingly straightforward tasks still remain beyond their ability.

"Computers are very good at calculation," says Atsuyuki Morishima, a professor at the Research Center for Knowledge Communities, "but ask them to identify whether a pair of images are similar or different, and they will have a hard time." In 2011, Morishima and his team combined the strengths of human and artificial intelligence in Crowd4U, a microtaskbased crowdsourcing tool tailored to the needs of academics.

Crowd4U divides a complex problem into thousands of simple tasks that human volunteers can perform within seconds. In the last three years, over 2,000 individuals have completed more than 200,000 tasks, such as assessing the condition of buildings in natural disaster areas and scanning tweets for nutrition-related

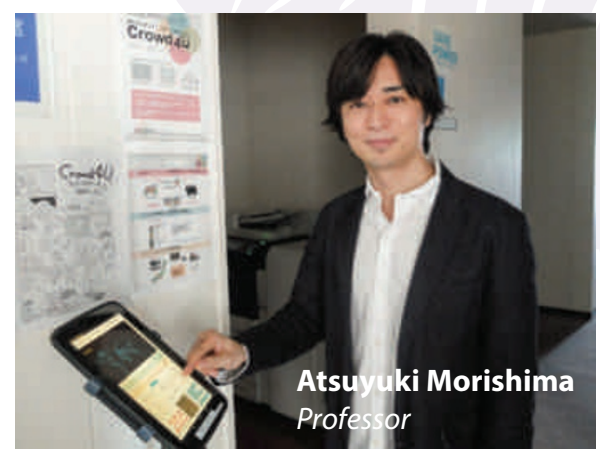

comments. "We can register virtually any kind of task in Crowd4U," says Morishima.

To facilitate the integration of the tasks into daily life, developers can make them pop up in smartphones or on the webpages of collaborating professors. Dedicated Crowd4U tablet stations have also been set up in seven universities across Japan, France and Belgium.

One of the nine ongoing projects on Crowd4U, known as L-Crowd, entails checking switches on and off target genes in response to environmental cues by changing the DNA structure. Therapies based on this process of mutation in epigenetic regulator genes are regarded by many as the future of medicine especially for cancer prevention and treatment.

TET2 has been found to be an important key in DNA demethylation - the process by which genes can be epigenetically activated or inactivated. "We know that this process is associated with aging and many cancers. So it is likely that TET2 mutation is the first step towards cancer, but the disease that eventually develops is actually determined by subsequent mutations," explains Chiba. "Why is this important? It has been reported that in fact 3\% of the healthy elderly population over 65 carry blood cells with the TET2 mutation. This mutation is frequently acquired with age, and may set the stage for the onset of blood cancers such as AITL as a result of mutations such as RHOA G17V. The results are promising, but there is still much to be discovered."

\section{Faculty of Medicine \\ \$www.md.tsukuba.ac.jp/top/en}

\section{We can register virtually any kind of task in Crowd4U.}

whether books recorded as having the same ISBN number in library databases across Japan are indeed the same. Any mismatches will be investigated later by researchers at the National Diet Library and several universities, who will 'clean' the erroneous records.

"Beyond solving problems, Crowd4U is also a useful tool for establishing the science of crowdsourcing," adds Morishima. It raises interesting questions about how to fragment a problem and consolidate the dispersed particles of information, in addition to offering a testing ground for improving the quality of crowdsourced data. "I really believe in the power of crowdsourcing and want to use it to make the world a better place."

Faculty of Library, Information and Media Science

-www.slis.tsukuba.ac.jp/grad/english/index-e.html 